\title{
Diffuse Gastric Polyposis: A Rare Case Report
}

\author{
Dr Abhijit S. Powar ${ }^{1}$, Dr Abhishek Kanbarkar ${ }^{2}$ \\ ${ }^{1}$ (Joy Hospital PVT. LTD. Mumbai/India) \\ ${ }^{2}$ (Joy Hospital PVT. LTD. Mumbai/India)
}

\begin{abstract}
In diffuse gastric polyposis, all or a large part of the gastric mucosa is covered with polyps. Diffuse gastric polyposis was accidentally found in a 45 years old male during study of his anaemia. The diagnosis was confirmed by oesophagogastroduodenoscopy and endoscopic biopsy. Very few cases reported those managed by laproscopically. We did a laparoscopic assisted distal gastrectomy with loop gastro gastrojejunostomy and feeding jejunostomy. Differential diagnosis of inherited gastrointestinal polyposis syndrome should be considered.malignant potential is the greast concern.
\end{abstract}

Keywords: Diffuse gastric polyposis, hyperplastic polyp.

\section{Introduction}

Gastric polyps can be broadly defined as luminal lesions projecting above the plane of the mucosal surface. Gastric polyposis is characterized by multiple gastric polyps. The term diffuse polyposis is used when all or a large part of the gastric mucosa is covered with polyps. We report a 45-year-old male with diffuse gastric polyposis found accidentally during study of his anemia. Gastric polyposis is also found in patients with various inherited gastrointestinal polyposis syndromes. Stomach manifestations were reviewed and differential diagnosis was considered. However, our patient did not fit into any of these polyposis syndromes. We also inspected all treatment modalities and finally, this patient underwent Laparoscopy-assisted distal gastrectomy (LADG) with less operative blood loss, less pain, earlier recovery of bowel activity, earlier resumption of oral intake, and a shorter hospital stay.

\section{Case report}

A 45-year male admitted to hospital with complaints of dyspnea with tiredness. On admission- Vital were stable. Physical examination revealed moderate built gentleman with pallor. On abdominal examination mild epigastric tenderness present .Per rectal examination was normal. The skin and mucosa had no pigmentation lesions, no palpable lymphadenopathy. Blood investigations showed hemoglobin $7.6 \mathrm{~g} / \mathrm{dl}$ and mean corpuscular volume $73 \mathrm{fL}$. Stool occult blood came positive. Other blood investigations came within normal. USG abdomen and CT abdomen were normal. Oesophagogastro-duodenoscopy was performed it revealed multiple sessile polypoidal lesions in distal body and antrum and in first part of duodenum. Biopsy of gastric polypoidal lesions showed features of benign hamartomatous polyp. . Colonoscopy study was within normal limits And there is no known family history of gastrointestinal tumor or inherited gastrointestinal polyposis syndrome . PET- CT showing presence of FDG avid large irregularly marginated soft tissue density in gastric pylorus extending into gastroduodenal junction causing luminal narrowing .Small and large bowel appear normal .

No significant mesenteric and retroperitoneal lymph node seen . In view of high FDG uptake HPE correlation is suggested considering neoplastic etiology. Under suspicion of diffuse gastric polyposis involving whole distal stomach and first part duodenum, laparoscopic assisted distal gastrectomy with duodenum distal to pylorus resected and loop gastro-jejunostomy done. And feeding jejunostomy done $30 \mathrm{~cm}$ distal to DJ junction.Intraoperatively No lymphadenopathy and liver, spleen, omentum were grossly normal .Post operatively jejunostomy tube feeding started with clear water and as tolerated gradually increased. After few days started soft diet orally. Patient tolerated orally and gradually intake increased. Patient recovered well postoperatively and continued to attend the clinic for check-ups. The consent to publish this case report was obtained from the patient.

Histopathology grossly mucosal surface showed multiple sessile and pedunculated polyps. Largest cauliflower like mass measured $5 \mathrm{~cm}$ in diameterMost polyps were soft and showed papillaroid and finely nodular surface. Microscopically polyps were hyperplastic and gastric fovolae showing branching and tufting. 


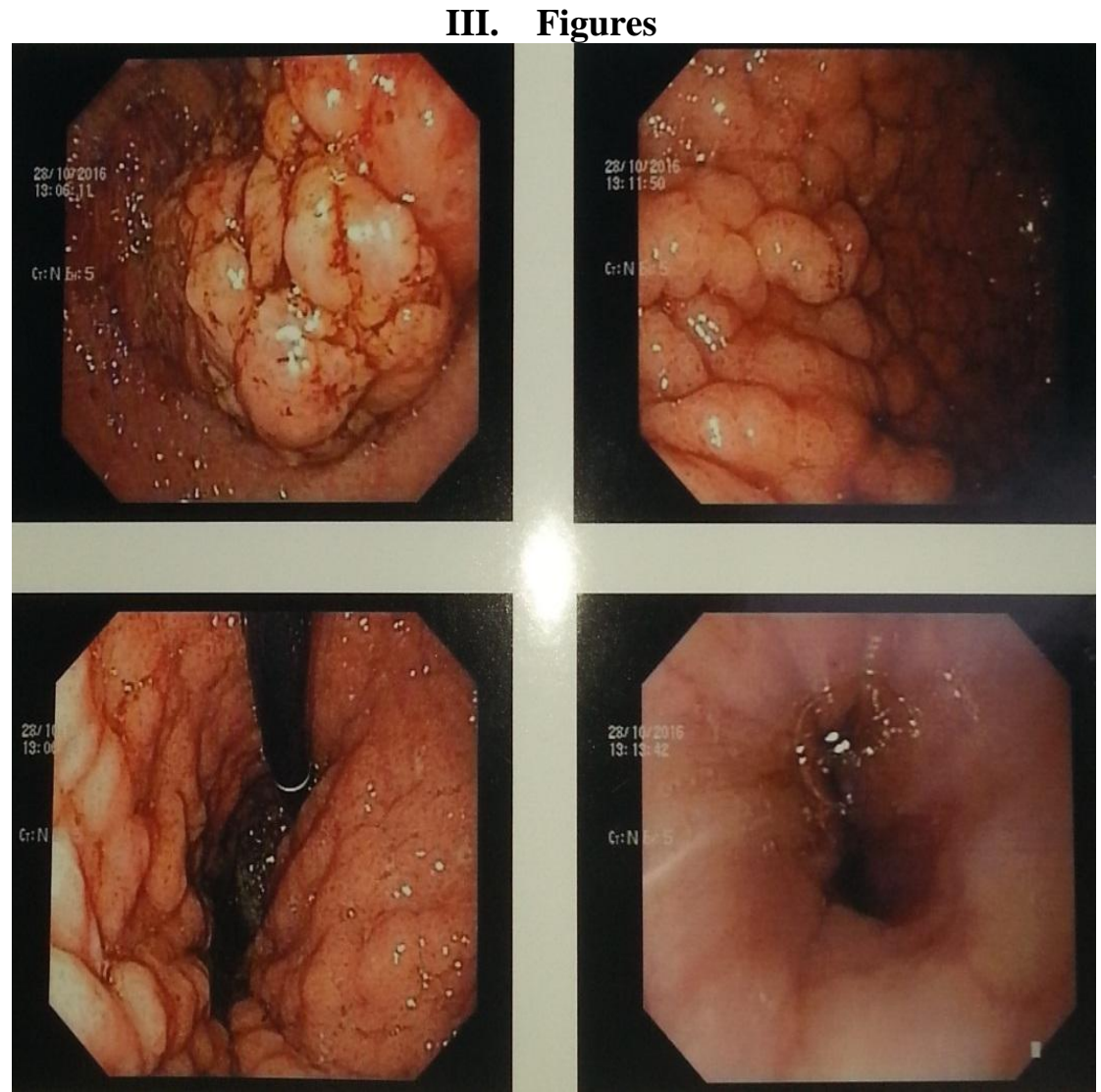

Fig 1. Oesophagogastroduodenoscopy showing multiple gastic polyp.

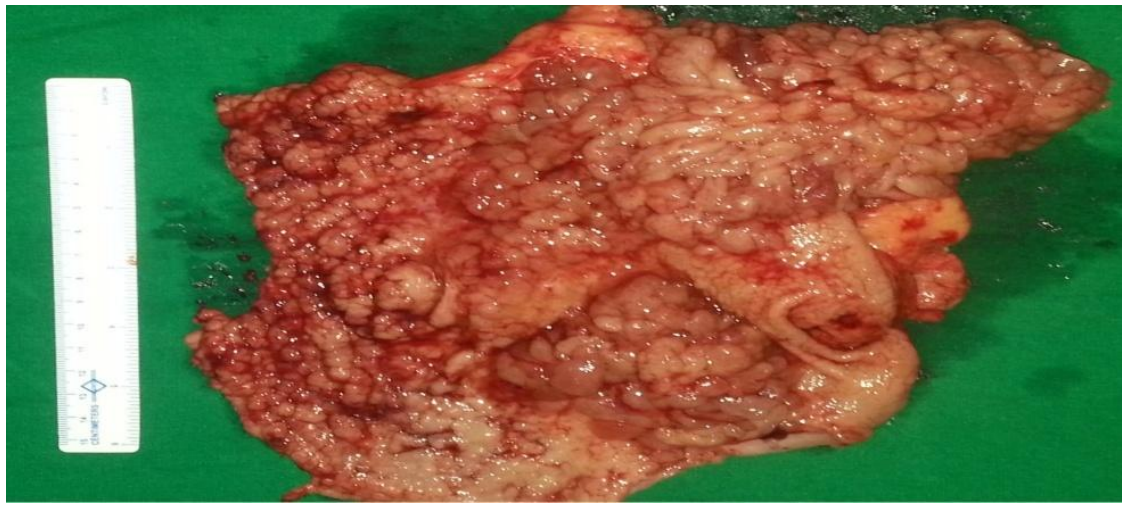

Fig 2: showing more than one third excised stomoch covered with polyps

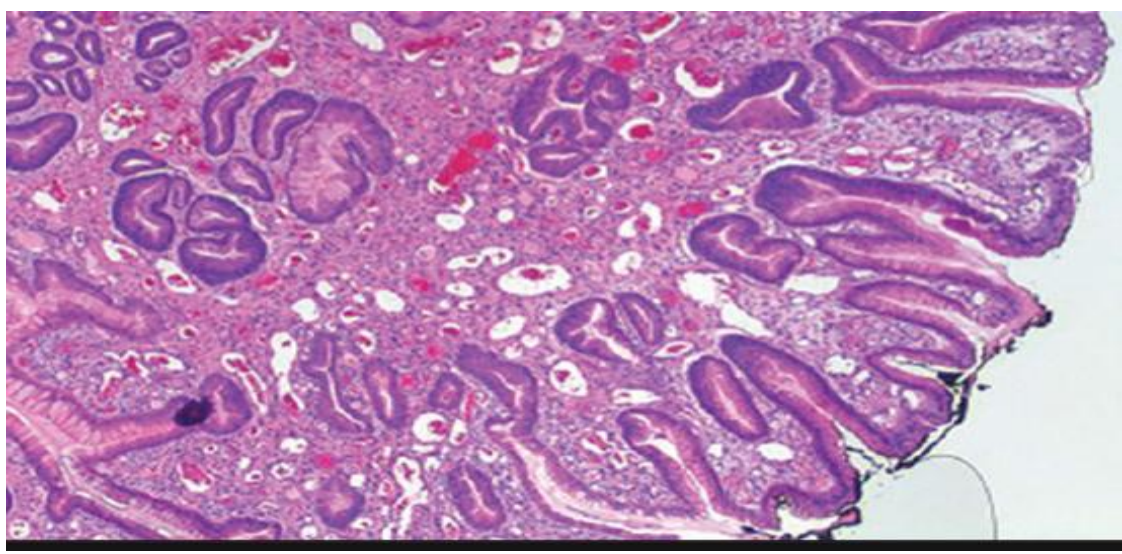

Fig 3 - hyperplastic polyp histopathology. 


\section{Discussion}

Gastric polyps are uncommon occurrence with incidence of less than 1\%.[1]. Some cases have been reported in Europe and Asia with variation in prevalence in different countries [2]. Patients with this type of lesions often present late with abdominal pain, palpable abdominal mass, haematemesis, anorexia, nausea and gastrointestinal bleeding. In our case, the tumour was not palpable and the patient was anaemic. This suggest that gastric inflammatory hyperplastic polyps may present with various signs and endoscopic examination remain important tool for identification of gastric tumours as it provides a minimally invasive approach to diagnosis and treatment [1]. Familial history of cancer can be considered and may be useful during diagnosis especially when this type of lesion is suspected in young aged patients. It is therefore important to take a detailed family history during diagnosis of gastric diseases especially in young aged patients. In our settings genetic investigations is not often done due financial limitations in of our patients. Thermal injury to the stomach seen in laser therapy for watermelon stomach can also give rise to gastric polyps [3]. Use of proton pump inhibitors, especially in children can give rise to gastric polyps and/or nodules [4]. Another interesting observation is the development of hyperplastic gastric polyps in patients who undergo solid organ transplantation and immunosuppressive therapy. The association of these polyps with the immunosuppressive therapy has not yet been well established [5].Our patient was not exposed to any immunosuppressive therapy and did not undergo any organ transplant.

Gastric polyps can be broadly defined as luminal lesions projecting above the plane of the mucosal surface.

They are relatively frequent in routine pathology practice, where the main goal is to rule out the possibility of malignancy. In diffuse gastric polyposis, which is rare, all or large part of gastric mucosa is covered with polyps. The rate of malignancy in these lesions can be high as $20 \%$, but maligncy is less common when the lesions are hyperplastic. [6,9] When evaluating patients who present with multiple gastrointestinal polyposis, many inherited polyposis syndromes should be considered in the differential diagnosis, although inpatients with most of these syndromes, there are few polyps in the stomach[10].The most common gastric lesion is fundic gland polyps or hamartoma, although hyperplastic polyps and adenomas have also been found[10]. Hyperplastic benign lesions are the second most common type of gastric polyp after fundic gland polyps[11-14] Upper gastrointestinal tract adenomas are well recognized complications of familial adenomatous polyposis (FAP).

In Gardner's syndrome, a clinical variant of FAP, cases of polyps in the small intestine and the stomach have been reported. The polyps were adenomatous, but far fewer in the stomach than in the small and large bowel. There have been no reports of cancer of the stomach in association with this syndrome[15]

Peutz-Jeghers syndrome is characterized by gastrointestinal polyposis and pigmentation. The polyposis is generalized, with the jejunum or ileum involved in nearly all patients, and the rectum, colon, stomach and duodenum less often. The malignant potential of hamartoma lesions is low, with a 2 to $3 \%$ risk of gastrointestinal cancer[16]

Juvenile polyposis also involves the whole of the gastrointestinal tract and is hamartomatous in nature, but polyps in the stomach are exceedingly rare and are thought to have no malignant potential. However, there are some reports that the malignant potential is low but definite[17].In our patient diffuse gastric polyposis was limited only to stomach and first part duodenum. Most reports of gastric polyposis do not differentiate between multiple and diffuse gastric polyposis, and there are generally less than five polyps in reports of multiple gastric polyposis. Case reports of sporadic diffuse gastric polyposis are few[10]. Malignancy is also more likely in patients with multiple polyps. In current practice, endoscopic removal for complete histologic examination constitutes adequate treatment.In our patient, the polyps covered a large area of the gastric mucosa and some were wide-based and sessile. This made total endoscopic removal impossible. Endoscopic follow-up carries the problem of inadequate histologic examination and an inability to declare the patient free of carcinomas.[10] Considering the current low mortality and morbidity of total gastrectomy, we decided to perform laproscopic assisted distal gastrectomy in this patient. We speculate that minimal manipulation of the intestine and the small incision required for LADG may prevent adhesion of the intestine and incisional hernia.[18] Undoubtedly, there are still several unsettled issues. Currently, we think this patient has sporadic diffuse gastric polyposis, but it is unclear if other family members will develop gastrointestinal tumors or if she will develop further gastrointestinal tract tumors Moreover, chronic gastritis and $H$. pylori infection were present in almost all of the patients with gastric polyposis[19,20] One can speculate that the inflammatory cell infiltration and acceleration of epithelial cell turnover induced by $H$. pylori infection contributes to the development and/or progression of hyperplastic polyps.

\section{Conclusion}

Isolated diffuse hyperplasic gastric polyposis is a rare entity and can present with severe anemia. Total gastrectomy or sub-total gastrectomy to prevent further occult blood loss and regular surveillance with endoscopy is necessary as there is a possibility of malignancy developing in these polyps. 


\section{References}

[1]. Jayawardena, S., Anandacoomaraswamy, D., Burzyantseva, O. \& Abdullah, M. (2008) Case Report: Isolated diffuse hyperplastic gastric polyposis presenting with severe anemia. Cases Journal 1, 1-3.

[2]. Carmack, S.W., Genta, R.M., Graham, D.Y. \& Lauwers, G.Y. (2009) Management of gastric polyps: a pathology based guide for gastroenterologists. Nature Reviews Gastroenterology \& Hepatology 6, 331-341.

[3]. Geller A, Gostout CJ, Balm RK: Development of hyperplastic polyps following laser therapy for watermelon stomach. Gastrointest Endosc 1996, 43(1):54-56.

[4]. Pashankar DS, Israel DM: Gastric polyps and nodules in children receiving long-term omeprazole therapy. J Pediatr Gastroenterol Nutr 2002, 35(5):658-662.

[5]. Amaro R, Neff GW, Karnam US, Tzakis AG, Raskin JB: Acquired hyperplastic gastric polyps in solid organ transplant patients. Am J Gastroenterol 2002, 97(9):2220-2224.

[6]. Marshak RH, Feldman F: Gastric polyps. Am J Dig Dis 1965;10:909-34.

[7]. Cornet A, Renault P, Carnot F: [Gastric polyps. Polyposis and chronic gastritis]. Arch Fr Mal App Dig 1971;60:507-24.

[8]. Weaver GA, Kleinman MS: Gastric polyposis due to multiple hyperplastic adenomatous polyps. Am J Dig Dis 1978; 23:346-52

[9]. Jagelman DG, DeCosse JJ, Bussey HJ: Upper gastrointestinal cancer in familial adenomatous polyposis. Lancet1988;i:1149-51.

[10]. Tai-Lin Hu, Jun-Te Hsu, Han-Ming Chen, and Miin-Fu ChenJFormos: diffuse gastric polyposis: report of a case Med Assoc2002;101:712-4)

[11]. Lindley PR, Appelman HD. Is there a preferred classification scheme for endoscopic gastric polyps? Mod Pathol. 1993;6:48A.

[12]. Seifert E, Gail K, Weismuller J. Gastric polypectomy: long-term results (survey of 23 centres in Germany). Endoscopy. 1983;15:811.

[13]. Stolte M, Sticht T, Eidt S, Ebert D, Finkenzeller G. Frequency, location, and age and sex distribution of various types of gastric polyp. Endoscopy. 1994;26: 659-665.

[14]. Laxen F, Sipponen P, Ihamaki T, Hakkiluoto A, Dortscheva Z. Gastric polyps; their morphological and endoscopical characteristics and relation to gastric carcinoma. Acta PatholMicrobiolImmunolScand [A]. 1982;90:221-228.

[15]. Bussey HJR: Gastrointestinal polyposis. Gut 1970;11:970-8.

[16]. Erbe RW. Inherited gastrointestinal-polyposis syndromes.NEngl J Med 1976;294:1101-4.

[17]. Watanabe A, Nagashima H, Motoi M, et al: Familial juvenile polyposis of the stomach. Gastroenterology 1979;77:148-51

[18]. Laparoscopy-Assisted Distal Gastrectomy for Early Gastric Cancer Is It Beneficial for Patients of Heavier Weight? Hirokazu Noshiro, MD, Shuji Shimizu, MD, Eishi Nagai, MD, Kenoki Ohuchida, MD, and Masao Tanaka, MD (.Ann Surg 2003;238:680685)

[19]. Seruca R, Carneiro F, Castedo S, David L, Lopes C, Sobrinho-Simões M. Familial gastric polyposis revisited: autosomal dominant inheritance confirmed. Cancer Genet Cytogenet 1991;53:97-100.

[20]. Carneiro F, David L, Seruca R, Castedo S, Nesland JM, Sobrinho-Simões M. Hyperplastic polyposis and diffuse carcinoma of the stomach: a study of a family. Cancer 1993;72:323-9. 